\title{
Neuropsychological Correlates of Transcription Factor AP-2Beta, and Its Interaction with COMT and MAOA in Healthy Females
}

\author{
Ina Schabrama ${ }^{a}$ Thomas Eggermann ${ }^{b}$ Steven J. Siegel ${ }^{c} \quad$ Gerhard Gründer $^{a}$ \\ Klaus Zerres $^{\mathrm{b}}$ Ingo Vernaleken ${ }^{\mathrm{a}}$ \\ a Department of Psychiatry and Psychotherapy, Medical Faculty, RWTH Aachen University, and JARA-Translational \\ Brain Medicine, and ${ }^{b}$ Institute of Human Genetics, RWTH Aachen University, Aachen, Germany; ${ }^{\mathrm{C}}$ Department of \\ Psychiatry Translational Research Laboratory, University of Pennsylvania, Philadelphia, Pa., USA
}

\section{Key Words}

AP-2 $\beta$ transcription factor $\cdot$ Catechol-O-methyltransferase . Monoamine oxidase A - Cognitive performance - Dopamine

\begin{abstract}
Background: The transcription factor AP- $2 \beta$ has been shown to impact clinical and neuropsychological properties. Apparently, it regulates the transcription of genes that code for molecules which are part of the catecholaminergic transmission system. This investigation focuses on possible effects of the transcription factor AP- $2 \beta$ intron 2 polymorphism on cognitive performance parameters. Methods: This hypothesis-driven investigation examined the effects and interactions of the transcription factor AP- $2 \beta$ intron 2 polymorphism, the Val158Met catechol-O-methyltransferase (COMT) polymorphism, and the variable number of tandem repeat polymorphism of monoamine oxidase A (MAOA) on cognitive performance parameters within a group of 200 healthy women (age: mean \pm SD, $23.93 \pm 3.33$ years). Results: The AP- $2 \beta$ polymorphism significantly influenced cognitive performance (in particular, the Trail Making Test part B), whereas the MAOA and COMT polymorphisms did not. However, there was an interaction effect of the AP- $2 \beta \times$ MAOA $\times C O M T$ genotypes on the decision bias $\beta$ of the degraded-stimulus version of the continuous performance task. Only the
\end{abstract}

Val158Met COMT polymorphism showed an influence on personality questionnaires (openness and self-transcendence; NEO Five-Factor Inventory, Temperament and Character Inventory). Conclusion: The transcription factor AP- $2 \beta$ intron 2 polymorphism had more influence on cognition than the MAOA and COMT polymorphisms. Possibly, the AP$2 \beta$ genotype might influence cognition through pathways other than those that regulate MAOA and COMT transcription. Interactions of transcription factor AP-2 $\beta, C O M T$, and MAOA polymorphisms suggest higher leverage effects of transcription factor AP- $2 \beta$ in subjects with high dopamine availability.

Copyright $\odot 2013$ S. Karger AG, Basel

\section{Introduction}

Transcription factors are proteins that regulate genetic transcription by binding to the promoter regions of genes. In the brain, the transcription factor AP- $2 \beta$ is widely involved in the synthesis of proteins, some of which are a part of the catecholaminergic and serotonergic transmission system. Accordingly, the synthesis of receptors, transporters, and enzymes within the dopamine system are affected by AP- $2 \beta$-mediated regulation. It has previously been reported that a polymorphic region of the

\begin{tabular}{ll}
\hline KARGER & $\begin{array}{l}\text { () 2013 S. Karger AG, Basel } \\
0302-282 X / 13 / 0682-0079 \$ 38.00 / 0 \quad \text { Karger }\end{array}$ \\
$\begin{array}{l}\text { E-Mail karger@karger.com } \\
\text { www.karger.com/nps }\end{array}$ & $\begin{array}{l}\text { This is an Open Access article licensed under the terms of the } \\
\text { Creative Commons Attribution-NonCommercial 3.0 Un- } \\
\text { ported license (CC BY-NC) (www.karger.com/OA-license), } \\
\text { applicable to the online version of the article only. Distribu- } \\
\text { tion permitted for non-commercial purposes only. }\end{array}$
\end{tabular}

Ingo Vernaleken

Department of Psychiatry, Psychotherapy, and Psychosomatics

RWTH Aachen University

Pauwelsstrasse 30, DE-52074 Aachen (Germany)

E-Mail ivernaleken@ukaachen.de 
AP- $2 \beta$ gene exists and that this region consists of a variable number of [CAAA] repeats in the second intron of the gene, which is located on chromosome 6p12-p21.1 [1]. This sequence is repeated either 4 or 5 times [2,3].

Previous studies have suggested that this AP- $2 \beta$ polymorphism is involved in several clinical and neurobiological parameters [4-6]. The AP- $2 \beta$ polymorphism is also associated with binge eating disorder; frequencies of homozygotic carriers of the long allele (5/5 [CAAA]) are higher in patients suffering from binge eating [7]. Furthermore, in healthy subjects, long-allele carriers exhibit lower scores on questionnaires that reflect anxiety-related personality traits and indirect aggression [1]. Finally, lower platelet monoamine oxidase $\mathrm{B}$ activity is associated with homozygote long-allele carrier status. In rats, the AP- $2 \beta$ genotype has been shown to have an impact on dopamine and serotonin metabolism in the frontal cortex [5]. Additionally, only females with 2 short alleles show decreased homovanillic acid (HVA) concentrations in the cerebrospinal fluid compared to carriers with at least 1 long allele [2]. These results suggest a substantial relevance of AP- $2 \beta$ in dopaminergic turnover and transmission. Other studies have indicated that a high dopamine synthesis capacity is supportive to maximize cognitive performance most probably by enabling an optimal modulation of dopamine signaling $[8,9]$. Following this line of reasoning, the AP- $2 \beta$ intron 2 polymorphism might be involved in individual cognitive performances. To the best of our knowledge, there are no previous studies that have investigated the influence of AP- $2 \beta$ on cognitive performance. We hypothesize that subjects with the $4 / 4$-repeat AP- $2 \beta$ genotype show better cognitive performance compared to $5 / 5$ carriers because the above-mentioned [2] decreased HVA levels in 4/4 carriers are thought to depict a lower dopamine metabolism by monoamine oxidase (MAO) enzymes, the formation of which is under control of AP- $2 \beta$. Under these premises, lower HVA levels might be a surrogate marker for higher capacities of dopamine transmission. The latter was consistently described to be associated with better cognitive performance [9-11].

Given the AP- $2 \beta$ intron 2 polymorphism would show a relevant effect on cognition via modulations of the dopamine metabolism, it might be hypothesized that other well-described polymorphisms that influence the pathways of dopamine degradation could exert additional effects. Some investigations were performed that focused on a polymorphism of the enzyme catechol-O-methyltransferase (COMT, Val158Met polymorphism; rs4680); COMT is an important enzyme of dopamine metabolism (for a review, see Barnett et al. [12]). Together with MAO, it is responsible for the degradation of dopamine into HVA. With respect to the COMT polymorphism, some authors reported that the Val alleles contribute to states of lower cognitive performance due to higher metabolism rates. Whereas some groups propose that the trimodal distribution of COMT activity results in such differences of cognition, other authors reported no effects [13-15]. A meta-analysis of Barnett et al. [12] found no or very little effect of COMT on cognitive performance.

The well-known variable number of tandem repeat (VNTR) MAOA polymorphism might theoretically also modulate the cognitive performance by different enzyme activities and different capacities of the dopamine transmission system. Whereas some studies focused on the behavioral impact of the VNTR MAOA polymorphism, no relevant investigation has been performed on cognition yet. Before the background of recent research results, strong effects of these COMT and MAOA polymorphisms could not be anticipated. However, it might be possible that lower enzyme activities of MAOA and COMT (e.g. COMT [Met/Met] and MAOA [3/3 VNTR]) could sum up to the postulated AP- $2 \beta$ effects (see before). Therefore, the proposed functional interplay between the AP-2 $\beta$, COMT, and MAOA polymorphisms was investigated in a sample of 200 healthy female subjects. All subjects were genetically characterized and performed cognitive tasks which were previously shown to be sensitive to dopaminergic transmission.

\section{Methods}

Subjects

This study was approved by the Ethics Committee of the Medical Faculty of RWTH Aachen University. All subjects were recruited by advertisements in public institutions. A total of 200 unrelated healthy females were included in the study after they had given written informed consent to participate. The mean age \pm SD was $23.93 \pm 3.33$ years. After an initial screening of 306 subjects by telephone conversation, 206 subjects were invited to attend the initial examination/visit (study information, check for inclusion/ exclusion criteria). The major exclusion criteria were mental disorders (according to the Diagnostic and Statistical Manual of Mental Disorders, fourth edition, including nicotine dependence/ abuse), somatic disorders, and the psychiatric treatment of firstdegree relatives. The inclusion criteria consisted of Caucasian origin, right-handedness, nonsmoker status, and age between 20 and 35 years. Six subjects failed to meet the inclusion criteria and were therefore excluded. The remaining 200 subjects underwent a neuropsychological test battery. An EDTA-anticoagulated blood sample $(10 \mathrm{ml})$ was drawn from each subject for genotype analysis.
80

Neuropsychobiology 2013;68:79-90 DOI: $10.1159 / 000350997$
Schabram/Eggermann/Siegel/Gründer/ Zerres/Vernaleken 


\section{Cognitive Assessment}

The primary intention of the cognitive test battery was to investigate the individual's prefrontal executive function, including sustained/divided attention, set-shifting capability, working memory, and psychomotor speed. This was based on previous investigations that successfully correlated the in vivo parameters of dopamine synthesis capacity with individual cognitive performance results [9]. We used the Stroop test [16], the Trail Making Test part B (TMT-B) [17], and a degraded-stimulus version of the continuous performance task (dsCPT) in combination with signal detection theory (adapted from Kathmann et al. [18]). When performing the latter test, 360 pictures were presented on a 21 -inch screen. A total of 180 of these pictures showed a well-perceptible cross (' + '), and 180 pictures showed the digits ' 0 '-' 9 '. The picture representing the ' 0 ' was defined as the target digit. Half of the 180 digits had well-perceptible contours, whereas the other half of the digits were presented with markedly blurred contours. Each digit was followed by a cross. The sequence of digits was randomly assigned with respect to the choice of digit ('0'-' 9 ') and their perceptibility (blurred vs. clear). After every presentation, an interstimulus interval of $1 \mathrm{~s}$ was interposed. The subject was instructed to press a button (independently from the sharpness) as fast as possible every time the digit ' 0 ' or a cross (' + ') was presented. The pictures were shown in blocks of 120 (60 digits and 60 crosses) presentations with 15 -second breaks; the complete test took $12 \mathrm{~min}$. The main outcome parameter was the percentage of correctly identified blurred ' 0 ' digits. Crosses and nonblurred ' 0 ' digits were included only for the validation of the subject's compliance.

The dsCPT was analyzed according to the signal detection theory [19]. Therefore, the parameters for hits, missed false alarms, and correct rejections were entered into the analysis using the BayesSDT software package for Matlab [20].

\section{Personality Assessment}

We additionally examined the personality traits of the subjects with the German version of the NEO Five-Factor Inventory (FFI) [21] and the short version of the Temperament and Character Inventory (TCI) [22].

\section{Genotyping}

Genomic DNA was isolated from peripheral lymphocytes with a routine salting-out procedure. For the determination of the AP$2 \beta$ and MAOA genotypes, standard polymerase chain reaction (PCR) amplifications were performed in a $25-\mu$ l volume containing $80 \mathrm{ng}$ of genomic DNA, 1 unit of recombinant Taq polymerase (Invitrogen, Germany), PCR buffer ( $10 \mathrm{~mm}$ Tris- $\mathrm{HCl}, 50 \mathrm{mM} \mathrm{KCl}$, $2.5 \mathrm{mM} \mathrm{MgCl}_{2}, \mathrm{pH} 8.3$ ), $200 \mathrm{mM}$ dNTPs, and $20 \mathrm{pmol}$ of each primer. The AP- $2 \beta$ primer sequences were obtained from Damberg et al. [1], and the MAOA primers were obtained from Sabol et al. [23]. For both variants, the forward primers were carboxyfluorescein-labeled. The PCR was run on an MJ PTC200 Temperature Cycler (Biozym, Germany), and each of the 35 cycles consisted of a $95^{\circ} \mathrm{C}$ denaturation step for $45 \mathrm{~s}$, a $63^{\circ} \mathrm{C}(\mathrm{AP}-2 \beta) / 62^{\circ} \mathrm{C}$ (MAOA) annealing step for $30 \mathrm{~s}$, and, finally, a $72^{\circ} \mathrm{C}$ elongation step for $90 \mathrm{~s}$. PCR products were run on an automated sequencing system (AB3130, Applied Biosystems, USA), and the electropherograms were analyzed with gene mapping software (Applied Biosystems, USA).

The COMT Val158Met genotypes were determined with a pyrosequencing assay. The region of interest was amplified using the
Table 1. Group characteristics regarding AP- $2 \beta, \mathrm{MAOA}$, and COMT genotypes

\begin{tabular}{llll}
\hline AP-2 $\beta$ & $4 / 4$ [CAAA, $\mathrm{n}$ & $4 / 5$ [CAAA], & $5 / 5$ [CAAA], \\
\hline & $24(12 \%)$ & $106(53 \%)$ & $69(34.5 \%)$ \\
\hline MAOA & High, $n$ & Medium, n & Low, $\mathrm{n}$ \\
\hline & $91(45.5 \%)$ & $90(45.0 \%)$ & $19(9.5 \%)$ \\
\hline COMT & Met/Met, $\mathrm{n}$ & Val/Met, $\mathrm{n}$ & $\mathrm{Val} / \mathrm{Val}, \mathrm{n}$ \\
\hline & $67(33.5 \%)$ & $94(47 \%)$ & $39(19.5 \%)$ \\
\hline
\end{tabular}

Distribution of AP-2 $\beta$, MAOA, and COMT genotypes in the group of 200 female subjects. MAOA-low alleles $=3 / 3$; MAOAmedium alleles $=3 / 3.5$ and $3 / 4 ;$ MAOA-high alleles $=3.5 / 3.5$ and $4 / 4$.

same standard PCR conditions as those described above. Primer sequences were the following: COMT_Pyro_F ( $5^{\prime}$-CCCAGCGGATGGTGGATTT-3') and COMT_Pyro_S (5'-GCGGATGGTGGATTT- $\left.3^{\prime}\right)$. The PCR amplification was performed as described for the AP-2 $\beta$ analysis, except the annealing temperature was $57^{\circ} \mathrm{C}$. After amplification, single strands were prepared with the Vacuum Prep Tool (Biotage, Uppsala, Sweden), which was followed by a denaturation step at $85^{\circ} \mathrm{C}$ for $2 \mathrm{~min}$ and the final sequencing primer hybridization (primer: COMT_Pyro_R: biotin$5^{\prime}$-CCTTTTTCCAGGTCTGACAACG-3'). Pyrosequencing was conducted with the PyroMark Gold Q96 reagents kit (Biotage AB).

Based on the results of Damberg et al. [1], we genetically defined the following 3 subgroups according to the individual [CAAA] repeat status of AP-2 $\beta$ : the homozygote 4/4-repeat allele combination (short alleles), the heterozygote $4 / 5$-repeat allele group, and the homozygote 5/5-repeat allele carriers (long allele). Additionally, the subjects were subdivided into high-, medium-, or lowactivity groups for the VNTR MAOA polymorphism and the COMT polymorphism. For the VNTR MAOA genotype, 3/3-repeat homozygotes were designated as low-activity, $3 / 3.5$ or $3 / 4$ heterozygotes were designated as medium-activity, and genotypes with 2 copies of the 3.5- or 4-repeat alleles were designated as highactivity subjects. The COMT Val158Met allele combination Met/ Met was designated as low activity, the $\mathrm{Val} / \mathrm{Met}$ combination was designated as medium, and the $\mathrm{Val} / \mathrm{Val}$ allele combination was designated as high activity.

\section{Statistical Analyses}

In order to investigate the main effects of the 3 genotypes (AP$2 \beta$, COMT, and MAOA) on cognition and personality, one-way ANOVAs were performed on the mean differences between the high-, medium-, and low-activity groups. In cases exhibiting significant results, a Sidak post hoc analysis was performed. In order to correct for multiple testing with respect to the number of cognitive tasks (dsCPT, TMT-B, Stroop), a Bonferroni correction was also performed: $p$ values less than 0.016 were considered to be statistically significant. 
Table 2. One-way ANOVA of the cognitive performance and AP- $2 \beta$, MAOA and COMT

\begin{tabular}{|c|c|c|c|c|c|c|c|}
\hline \multicolumn{5}{|c|}{ Measure } & \multirow[t]{2}{*}{$\mathrm{F}$} & \multicolumn{2}{|c|}{ d.f. $p$ value } \\
\hline \multirow[t]{5}{*}{ MAOA } & & high & medium & low & & & \\
\hline & TMT-B, s & $53.37(91)$ & $46.38(90)$ & $47.26(19)$ & 2.296 & 2 & 0.103 \\
\hline & Stroop interference, $s$ & $68.56(91)$ & $63.01(90)$ & $65.49(19)$ & 2.141 & 2 & 0.120 \\
\hline & dsCPT hits & $6.31(91)$ & $5.68(89)$ & $5.71(19)$ & 0.576 & 2 & 0.563 \\
\hline & Decision bias $\beta$ & $0.08(91)$ & $0.34(89)$ & $0.36(19)$ & 1.484 & 2 & 0.229 \\
\hline \multirow[t]{5}{*}{ COMT } & & Met/Met & Val/Met & $\mathrm{Val} / \mathrm{Val}$ & & & \\
\hline & TMT-B, s & $49.81(67)$ & $46.70(94)$ & $45.18(39)$ & 1.852 & 2 & 0.160 \\
\hline & Stroop interference, $s$ & $64.96(67)$ & $64.67(94)$ & $64.13(39)$ & 0.059 & 2 & 0.943 \\
\hline & dsCPT hits & $5.91(67)$ & $5.56(93)$ & $5.97(39)$ & 0.620 & 2 & 0.539 \\
\hline & Decision bias $\beta$ & $0.30(67)$ & $0.39(93)$ & $0.22(39)$ & 0.947 & 2 & 0.390 \\
\hline \multirow[t]{5}{*}{ AP- $2 \beta$} & & $4 / 4$ [CAAA] & 4/5 [CAAA] & $5 / 5$ [CAAA] & & & \\
\hline & TMT-B, s & $40.33(24)$ & $47.62(106)$ & $49.77(69)$ & 4.831 & 2 & $0.009^{* *}$ \\
\hline & Stroop interference, $s$ & $62.63(24)$ & $65.03(106)$ & $64.55(69)$ & 0.40 & 2 & 0.671 \\
\hline & dsCPT hits & $5.62(24)$ & $5.78(105)$ & $5.84(69)$ & 0.073 & 2 & 0.929 \\
\hline & Decision bias $\beta$ & $0.55(24)$ & $0.29(105)$ & $0.31(69)$ & 1.461 & 2 & 0.235 \\
\hline
\end{tabular}

Results of the ANOVA for AP-2 $\beta$, MAOA and COMT and the neuropsychological test battery, which consists of TMT-B, Stroop interference, dsCPT hit rate, and the decision bias $\beta$ resulting from the signal detection theory analysis of the dsCPT. MAOA-low alleles $=3 / 3$, MAOA-medium alleles $=3 / 3.5$ and 3/4, and MAOA-high alleles 3.5/3.5 and $4 / 4 .{ }^{* *} \mathrm{p}<0.01$. Figures in parentheses indicate numbers.

In order to analyze the interactions of the 3 polymorphisms, a generalized linear model was applied for all of the main outcome variables for the Stroop, dsCPT hits, decision bias ( $\beta$ ), and TMT-B tests. The model included the 3 genetic main effects as predictors, age as a covariate, and the 2 gene $\times$ gene interaction terms.

The Hardy-Weinberg equilibrium was analyzed with a web tool by Rodriguez et al. [24].

\section{Results}

\section{Gene Frequencies}

The allele frequencies for the variable number of AP$2 \beta$ [CAAA] sequences were $38.5 \%(n=154)$ for $4 \times$ [CAAA] and $61.3 \%(\mathrm{n}=245)$ for $5 \times$ [CAAA], and there was 1 rare variation $(0.3 \%)$. The VNTR MAOA polymorphism allele frequencies were 3-repeat [31.8\% $(\mathrm{n}=127)]$, 3.5 -repeat $[1.8 \%(\mathrm{n}=7)]$, 4-repeat $[65.3 \%(\mathrm{n}=261)]$, and 5 -repeat $[1.3 \%(n=5)]$. The allele frequencies for the COMT Val158Met genotypes were $43 \%(\mathrm{n}=172)$ for the Val allele and 57\% $(n=228)$ for the Met allele. See table 1 for the group frequencies of the polymorphisms or genetic groups, respectively. All polymorphisms were in
Hardy-Weinberg equilibrium (AP- $2 \beta: \chi^{2}=3, \mathrm{p}>0.05$; MAOA: $\chi^{2}=0.34, \mathrm{p}>0.05$; COMT: $\chi^{2}=0.34, \mathrm{p}>0.05$ ). No significant differences were found for age or educational level in the 3 genetic groups.

\section{Cognitive Measurements}

The one-way ANOVA for the AP- $2 \beta$ polymorphism revealed a significant main effect on the TMT-B results $(\mathrm{F}=4.831$, d.f. $=2, \mathrm{p}=0.009, \mathrm{n}=199$; table 2$)$. The Sidak post hoc analyses revealed that the $4 / 4(40.33 \pm 9.01)$ carriers performed better than the 5/5 carriers (49.77 \pm 11.29; $\mathrm{p}=0.007)$ and the $4 / 5$ carriers $(47.62 \pm 14.39 ; \mathrm{p}=$ 0.038 ) (for details, see table 3 and fig. 1). The other cognitive tests did not show any significant group differences. The group difference between $4 / 4$ and 5/5 carriers survived Bonferroni correction for the inclusion of multiple cognitive tasks.

The MAOA and COMT polymorphisms exhibited no significant effects on the performances of the cognitive tasks in the present sample. 
Table 3. Sidak post hoc analyses for the cognitive tasks and AP-2 $\beta$, MAOA and COMT

\begin{tabular}{|c|c|c|c|c|c|c|}
\hline \multirow[t]{2}{*}{ AP- $2 \beta$} & \multicolumn{2}{|l|}{ Group 4/4-5/5 } & \multicolumn{2}{|l|}{ Group 4/4-4/5 } & \multicolumn{2}{|l|}{ Group 4/5-5/5 } \\
\hline & mean difference & $\mathrm{p}$ & mean difference & $\mathrm{p}$ & mean difference & $\mathrm{p}$ \\
\hline TMT-B & -9.435 & $0.007^{* *}$ & -7.289 & $0.038^{*}$ & -2.145 & 0.628 \\
\hline Stroop interference & n.s. & n.s. & n.s. & n.s. & n.s. & n.s. \\
\hline dsCPT hits & n.s. & n.s. & n.s. & n.s. & n.s. & n.s. \\
\hline Decision bias $\beta$ & n.s. & n.s. & n.s. & n.s. & n.s. & n.s. \\
\hline \multirow[t]{2}{*}{ COMT } & \multicolumn{2}{|c|}{ Group Met/Met-Val/Val } & \multicolumn{2}{|c|}{ Group Met/Met-Val/Met } & \multicolumn{2}{|c|}{ Group Val/Met-Val/Val } \\
\hline & mean difference & $\mathrm{p}$ & mean difference & $\mathrm{p}$ & mean difference & $\mathrm{p}$ \\
\hline TMT-B & n.s. & n.s. & n.s. & n.s. & n.s. & n.s. \\
\hline Stroop interference & n.s. & n.s. & n.s. & n.s. & n.s. & n.s. \\
\hline dsCPT hits & n.s. & n.s. & n.s. & n.s. & n.s. & n.s. \\
\hline Decision bias $\beta$ & n.s. & n.s. & n.s. & n.s. & n.s. & n.s. \\
\hline \multirow[t]{2}{*}{ MAOA } & \multicolumn{2}{|l|}{ Group low-high } & \multicolumn{2}{|c|}{ Group low-middle } & \multicolumn{2}{|c|}{ Group middle-high } \\
\hline & mean difference & $\mathrm{p}$ & mean difference & $\mathrm{p}$ & mean difference & $\mathrm{p}$ \\
\hline \multirow{4}{*}{$\begin{array}{l}\text { TMT-B } \\
\text { Stroop interference } \\
\text { dsCPT hits } \\
\text { Decision bias } \beta\end{array}$} & n.s. & n.s. & n.s. & n.s. & n.s. & n.s. \\
\hline & n.s. & n.s. & n.s. & n.s. & n.s. & n.s. \\
\hline & n.s. & n.s. & n.s. & n.s. & n.s. & n.s. \\
\hline & n.s. & n.s. & n.s. & n.s. & n.s. & n.s. \\
\hline
\end{tabular}

Post hoc analyses for AP-2 $\beta$ MAOA and COMT and the neuropsychological test battery consisting of TMT$\mathrm{B}$, Stroop interference score, dsCPT hit rate, and the decision bias $\beta$ resulting from the signal detection theory analysis. MAOA-low alleles $=3 / 3$, MAOA-medium alleles $=3 / 3.5$ and 3/4, and MAOA-high alleles 3.5/3.5 and $4 / 4{ }^{*} \mathrm{p}<0.05 ;{ }^{* *} \mathrm{p}<0.01$; n.s. $=$ not significant.

\section{Personality Assessment}

The COMT polymorphism showed main effects on some dimensions of the NEO-FFI and TCI. The openness item in the NEO-FFI revealed a main group effect $(\mathrm{F}=$ 3.575 , d.f. $=2, \mathrm{p}=0.030, \mathrm{n}=198$ ). The corresponding post hoc analysis indicated that the Val/Val group showed significantly higher scores when compared to the $\mathrm{Val} / \mathrm{Met}$ group ( $\mathrm{p}=0.036$ ) (for results, see table 4) but not when compared to the Met/Met group. Within the TCI, the self-transcendence subscale was significantly influenced by COMT $(\mathrm{F}=3.329$, d.f. $=2, \mathrm{p}=0.038, \mathrm{n}=170)$. Further analysis showed that the Met/Met group had significantly lower scores than the $\mathrm{Val} / \mathrm{Val}$ group $(27.45 \pm 12.79 \mathrm{vs}$. $35.68 \pm 16.54, \mathrm{p}=0.033$ ) (table 5).

No significant effects on personality were found for the AP- $2 \beta$ and MAOA polymorphisms.

Furthermore, a negative correlation between decision bias $\beta$ and neuroticism of the NEO-FFI exists $(r=-1.46$, $\mathrm{p}=0.04)$; there were no significant correlations between $\beta$ and any further personality items.

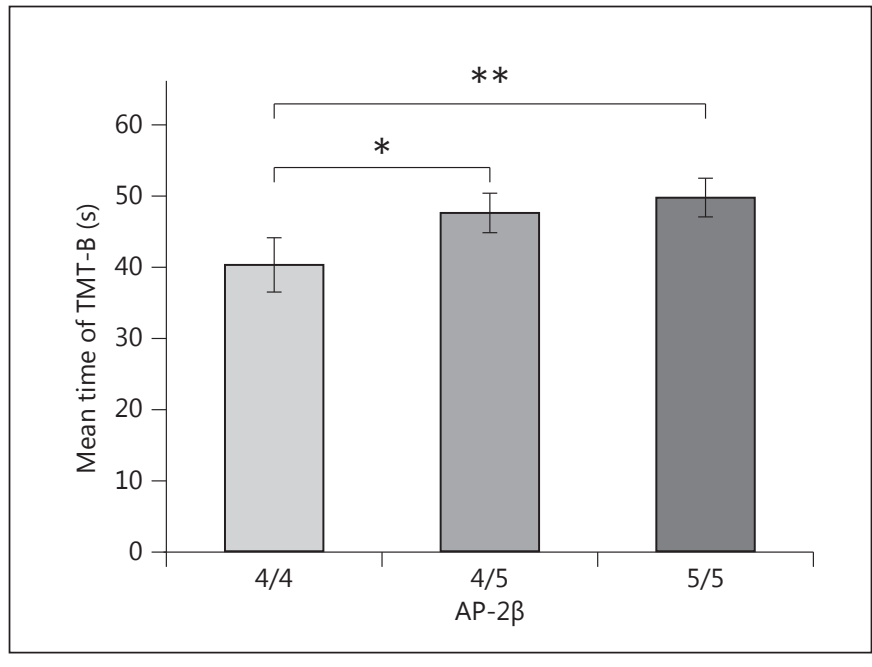

Fig. 1. AP- $2 \beta$ and TMT-B. Mean time of TMT-B in seconds. AP$2 \beta$ divided into groups based on the [CAAA] repetition. ${ }^{*} \mathrm{p}<0.05$; ${ }^{* *} \mathrm{p}<0.01$. Error bars: $95 \%$ confidence interval. 
Table 4. ANOVA of personality questionnaires and COMT

\begin{tabular}{lllllll}
\hline & COMT & & & & d.f. & p value \\
\cline { 2 - 4 } & Met/Met & Val/Met & Val/Val & & & \\
\hline NEO-Five-Factor Inventory & $(\mathrm{n}=66)$ & $(\mathrm{n}=94)$ & $(\mathrm{n}=38)$ & & & \\
Neuroticism & 1.41 & 1.26 & 1.31 & 1.577 & 2 & 0.209 \\
Extraversion & 2.62 & 2.64 & 2.53 & 0.710 & 2 & 0.493 \\
Openness & 2.69 & 2.57 & 2.78 & 3.575 & 2 & $0.030^{*}$ \\
Agreeableness & 2.88 & 2.92 & 2.86 & 0.334 & 2 & 0.717 \\
Conscientiousness & 2.79 & 2.82 & 2.84 & 0.082 & 2 & 0.921 \\
\hline Temperament and Character Inventory & $(\mathrm{n}=54)$ & $(\mathrm{n}=81)$ & $(\mathrm{n}=35)$ & & & \\
Novelty seeking & 41.37 & 39.42 & 37.03 & 2.406 & 2 & 0.093 \\
Harm avoidance & 30.74 & 30.99 & 35.74 & 1.611 & 2 & 0.203 \\
Reward dependence & 51.44 & 52.14 & 50.00 & 0.555 & 2 & 0.575 \\
Persistence & 46.39 & 48.99 & 48.06 & 0.976 & 2 & 0.379 \\
Self-directedness & 59.72 & 63.17 & 60.94 & 1.769 & 2 & 0.174 \\
Cooperativeness & 64.96 & 65.25 & 62.94 & 0.870 & 2 & 0.421 \\
Self-transcendence & 27.45 & 31.38 & 35.68 & 3.329 & 2 & $0.038^{*}$ \\
\hline \multicolumn{1}{c}{$*$ p $<$ 0.05. } & & & & & & \\
\hline
\end{tabular}

Table 5. Results of the Sidak post hoc test for TCI and COMT

\begin{tabular}{|c|c|c|c|c|c|c|}
\hline \multirow[t]{3}{*}{ TCI } & \multicolumn{6}{|l|}{ COMT } \\
\hline & \multicolumn{2}{|c|}{ group Met/Met vs. Val/Val } & \multicolumn{2}{|c|}{ group Met/Met vs. Val/Met } & \multicolumn{2}{|c|}{ group Val/Met vs. Val/Val } \\
\hline & mean difference & $\mathrm{p}$ & mean difference & $\mathrm{p}$ & mean difference & $\mathrm{p}$ \\
\hline Novelty seeking & n.s. & n.s. & n.s. & n.s. & n.s. & n.s. \\
\hline Harm avoidance & n.s. & n.s. & n.s. & n.s. & n.s. & n.s. \\
\hline Reward dependence & n.s. & n.s. & n.s. & n.s. & n.s. & n.s. \\
\hline Persistence & n.s. & n.s. & n.s. & n.s. & n.s. & n.s. \\
\hline Self-directedness & n.s. & n.s. & n.s. & n.s. & n.s. & n.s. \\
\hline Cooperativeness & n.s. & n.s. & n.s. & n.s. & n.s. & n.s. \\
\hline Self-transcendence & -8.22 & $0.033^{*}$ & -3.92 & 0.349 & 4.30 & 0.390 \\
\hline
\end{tabular}

n.s. $=$ Not significant; ${ }^{*} \mathrm{p}<0.05$.

\section{Interactions}

In order to investigate the possible interactions between the genes, the main effects of the AP- $2 \beta$, MAOA, and COMT genotypes, as well as the interaction terms AP- $2 \beta \times$ MAOA, AP $-2 \beta \times$ COMT, and AP- $2 \beta \times$ MAOA $\times$ COMT, with age as a scaled covariate, were tested in a generalized linear model. Highly significant main effects were found for all 3 polymorphisms: AP- $2 \beta$, MAOA, and $\operatorname{COMT}\left(\chi^{2}=7.466, \mathrm{p}=0.024 ; \chi^{2}=11.675, \mathrm{p}=0.003 ; \chi^{2}=\right.$ 9.111, $\mathrm{p}=0.011$, respectively). Furthermore, there were interaction effects of AP- $2 \beta$ and MAOA and for AP- $2 \beta$,
MAOA, and COMT $\left(\chi^{2}=12.044, \mathrm{p}=0.017 ; \chi^{2}=25.923\right.$, $\mathrm{p}=0.004$, respectively; table 6). Post hoc $\mathrm{t}$ tests for the AP- $2 \beta \times$ MAOA interaction showed that, in the highactivity MAOA group, a significant difference in decision bias existed between the $4 / 4$ AP- $2 \beta$ carriers and the $4 / 5$ carriers $(\mathrm{t}=2.431, \mathrm{p}=0.018)$. The $4 / 4$ carriers showed a more conservative bias, whereas the $4 / 5$ carriers showed only a minimally conservative bias. Due to the genetic distribution and allele frequencies, some genetic interaction clusters consisted of less than 2 subjects (see fig. 3 for AP- $2 \beta \times$ MAOA interaction). No reasonable inter- 
Table 6. Generalized linear model analysis for the neuropsychological tests: relationship between AP-2 $\beta$, MAOA and COMT

\begin{tabular}{|c|c|c|c|c|c|c|c|c|c|c|c|c|}
\hline \multicolumn{13}{|l|}{ Main effects } \\
\hline AP- $2 \beta$ & 3.692 & 2 & 0.158 & 0.367 & 2 & 0.832 & 0.689 & 2 & 0.709 & 9.765 & 2 & 0.008 \\
\hline MAOA & 1.774 & 2 & 0.412 & 2.378 & 2 & 0.304 & 2.588 & 2 & 0.274 & 14.595 & 2 & $0.001^{* *}$ \\
\hline COMT & 0.020 & 2 & 0.990 & 0.466 & 2 & 0.792 & 4.027 & 2 & 0.134 & 11.453 & 2 & $0.003^{* *}$ \\
\hline $\mathrm{AP}-2 \beta \times \mathrm{MAOA}$ & 0.920 & 4 & 0.922 & 4.596 & 4 & 0.331 & 2.515 & 4 & 0.642 & 9.988 & 4 & 0.041 \\
\hline $\mathrm{AP}-2 \beta \times \mathrm{COMT}$ & 6.833 & 4 & 0.145 & 6.447 & 4 & 0.168 & 5.58 & 4 & 0.233 & 7.462 & 4 & 0.113 \\
\hline $\mathrm{AP}-2 \beta \times \mathrm{MAOA} \times \mathrm{COMT}$ & 6.446 & 10 & 0.776 & 10.714 & 10 & 0.380 & 18.025 & 10 & 0.055 & 32.826 & 10 & $0.000^{* *}$ \\
\hline
\end{tabular}

Results of generalized linear model analysis of AP- $2 \beta$, MAOA and COMT and the interaction terms AP- $2 \beta \times \mathrm{MAOA}, \mathrm{AP}-2 \beta \times \mathrm{COMT}$ and $\mathrm{AP}-2 \beta \times \mathrm{MAOA} \times \mathrm{COMT}$ on the 4 neuropsychological tests TMT-B, Stroop interference, dsCPT hit rate and decision bias $\beta$. Age is included as covariate. ${ }^{* *} \mathrm{p}<0.01$.

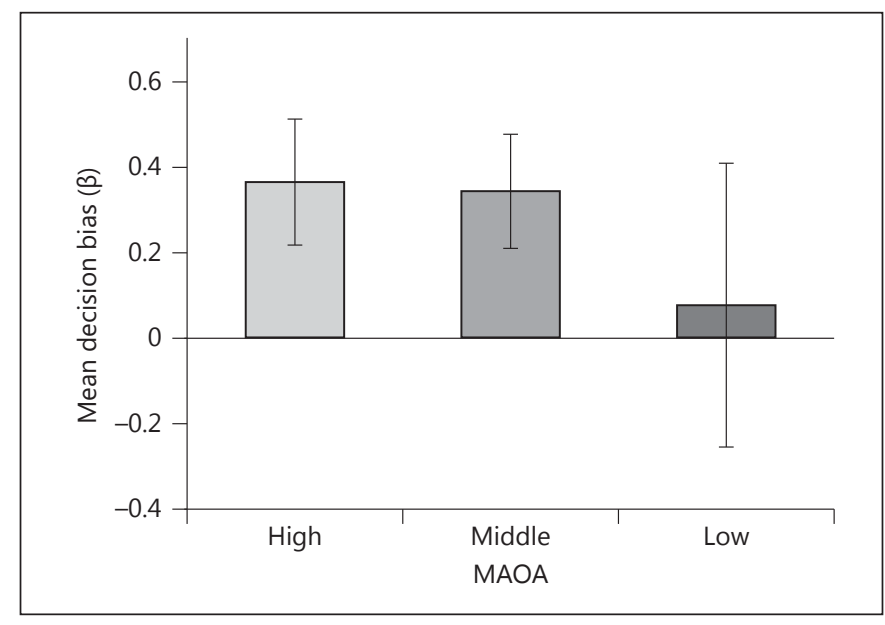

Fig. 2. MAOA and decision bias $\beta$. Results of VNTR MAOA on decision bias $\beta$. Positive $\beta$ : conservative bias; greater tendency to say 'no'. Negative $\beta$ : liberal bias; greater tendency to say 'yes'. Error bars: $95 \%$ confidence interval.

pretations can be drawn from these clusters. Nevertheless, these findings with respect to the decision bias encourage performing replication studies with larger sample.

The post hoc analyses for the AP- $2 \beta \times$ MAOA $\times$ COMT interaction revealed that there was a significant difference in the decision bias between the MAOA highactivity group $(\mathrm{MAOA}-\mathrm{H}) \times \mathrm{COMT}(\mathrm{Val} / \mathrm{Met}) \times 5 / 5 \mathrm{AP}-$ $2 \beta$ and the MAOA low-activity group (MAOA-L) $\times$

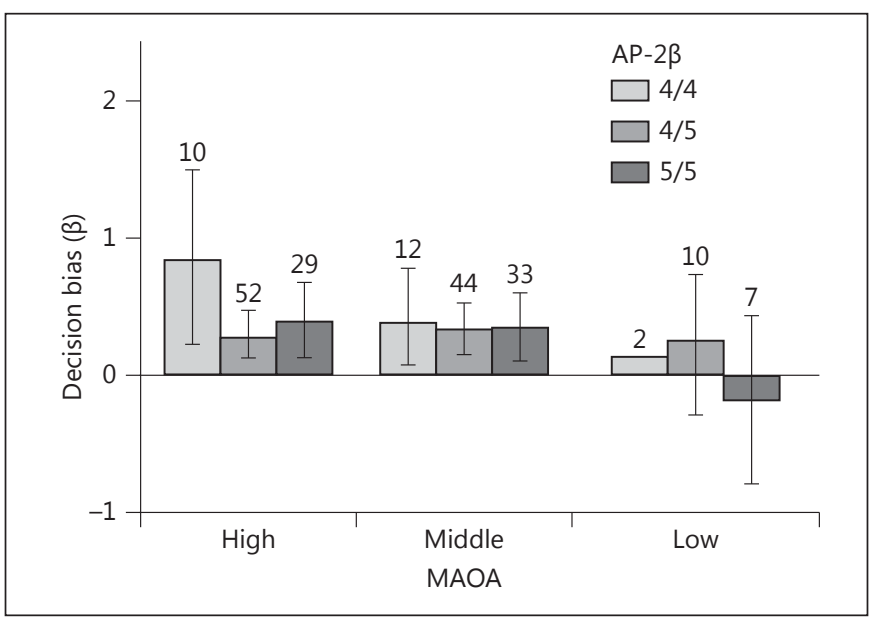

Fig. 3. Interaction of AP- $2 \beta \times$ MAOA on decision bias $\beta$. Results of the interaction of AP- $2 \beta$ and MAOA on decision bias $\beta$. Positive $\beta$ : conservative bias; greater tendency to say 'no'. Negative $\beta$ : liberal bias; greater tendency to say 'yes'. Error bars 2 SE. The digits above the error bars indicate the group sizes of the cluster. Error bars: $95 \%$ confidence interval.

COMT $(\mathrm{Val} / \mathrm{Met}) \times 5 / 5 \mathrm{AP}-2 \beta$, which showed that the MAOA-L group had a liberal bias, whereas the MAOA-H group showed a more conservative bias. Further post hoc tests exhibited a significant difference for the MAOA-H $\times \mathrm{COMT}(\mathrm{Val} / \mathrm{Met}$ ) group between the $4 / 5 \mathrm{AP}-2 \beta$ and $5 / 5$ AP- $2 \beta$ carriers $(t=-2.717, p=0.01)$, resulting in a more conservative bias for group $5 / 5$. The difference between the 4/5 and 5/5 carriers in the MAOA-L $\times$ COMT (Val/ Met) group showed an even stronger significance $(t=$ 


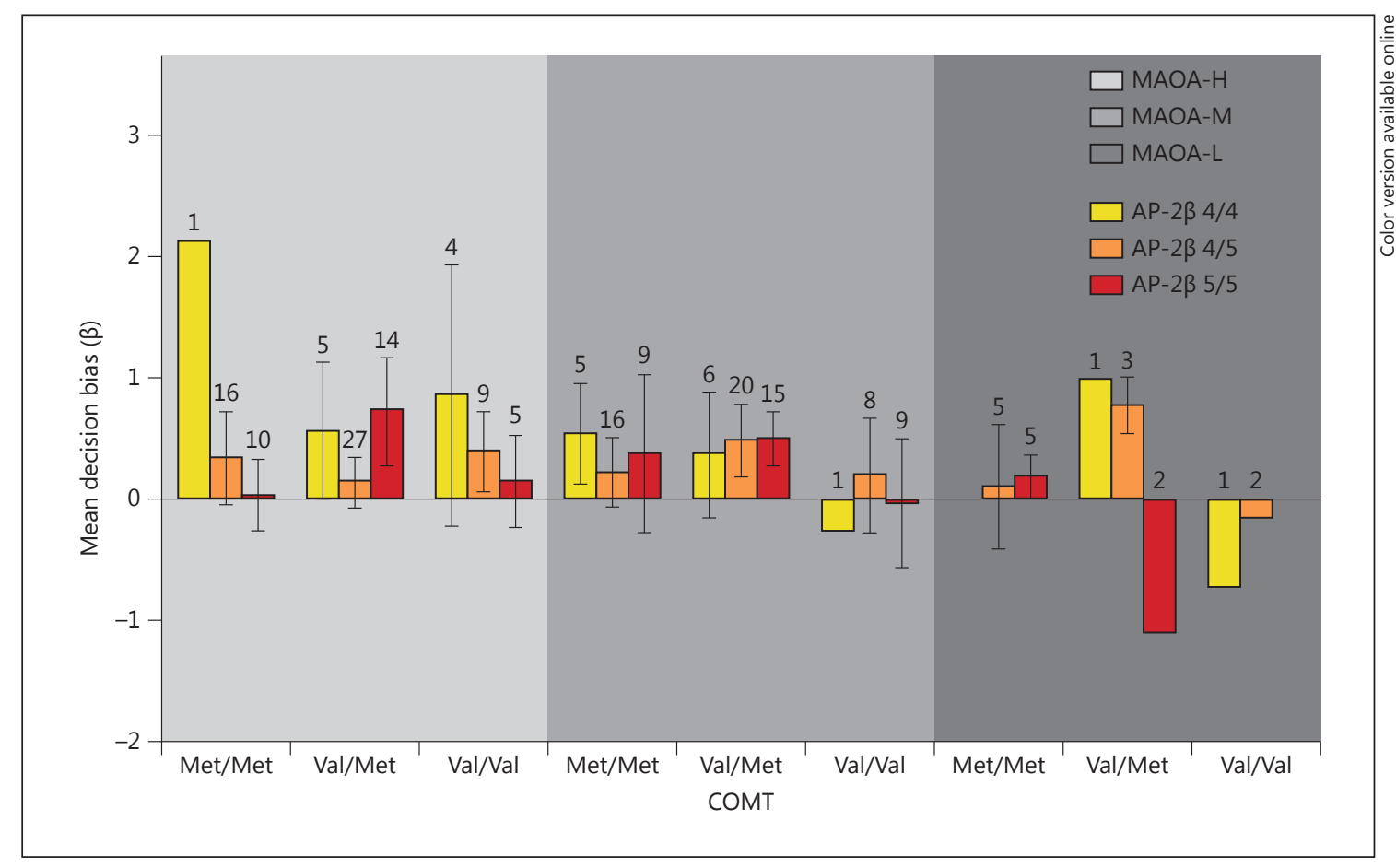

Fig. 4. Interaction of AP $-2 \beta \times$ MAOA $\times$ COMT on decision bias $\beta$. Results of the interaction of AP-2 $\beta$, MAOA, and COMT on decision bias $\beta$. Positive $\beta$ : conservative bias; greater tendency to say 'no'. Negative $\beta$ : liberal bias; greater tendency to say 'yes'. Error bars 2 SE. The digits above the error bars indicate the group sizes of the cluster. Error bars: 95\% confidence interval.

$8.636, \mathrm{p}=0.003)$. The $5 / 5$ carriers showed a liberal bias, whereas the $4 / 5$ carriers had a conservative bias. The MAOA-L $\times$ COMT $(\mathrm{Val} / \mathrm{Met}) \times 5 / 5 \mathrm{AP}-2 \beta$ group had a significantly more liberal bias than almost every other combination with the $5 / 5 \mathrm{Ap}-2 \beta$ allele (see fig. 4 for AP$2 \beta \times$ MAOA $\times$ COMT interaction).

Besides these effects on the decision bias, a borderlinesignificant interaction between AP-2 $\beta$, MAOA, and COMT for the hit rate of the dsCPT was shown $\left(\chi^{2}=\right.$ 18.025, d.f. $=10 ; p=0.055)$.

There were no effects for the TMT-B or the Stroop interference task. After very conservative correction for multiple testing, including all test modalities, assessments, and genotypes, no significant group differences could be observed.

\section{Discussion}

In the present study, we investigated the effects of the polymorphisms of AP-2 $\beta, \mathrm{MAOA}$, and COMT on the cognitive performance and personality dimensions of a sample of 200 healthy female subjects. The choices of the polymorphisms and the study protocol were strictly hypothesis-driven by the postulated tight functional interplay of their corresponding gene products on the metabolism of catecholamines.

The main finding was a positive group effect of the AP- $2 \beta$ genotype on the TMT-B, which is a task that previously showed robust associations with dopaminergic in vivo parameters [9]. The corresponding post hoc analysis indicated that those individuals with 2 short alleles performed better on the TMT-B than the heterozygote and homozygote long-allele subjects. Interestingly, the group effects of the MAOA promoter polymorphism, as well as the rs4680 COMT polymorphism, showed far less pronounced and nonstatistically significant associations with cognitive performance, even though several (but not all) previous investigations reported a reliable influence of these genotypes on catecholaminergic metabolism [12, 13, 25]. Nevertheless, Damberg et al. [2] observed that females who are homozygotic for the short allele showed lower HVA levels than subjects with at least 1 long allele. Thus, the AP- $2 \beta$ genotype appears to be 
functionally relevant to dopamine metabolism, potentially by modulating the transcription of the MAOA gene. Indeed, most of the previous investigations have reported a positive association of HVA concentrations and cognitive outcome parameters. This has mainly been interpreted to be caused by variations in dopamine synthesis. An AP-2 $\beta$-associated reduction of MAOA [2] and COMT transcription might reduce dopamine metabolism, resulting in lower dopamine turnover rates and increased dopamine availability because less dopamine is degraded into HVA. In general, most healthy subjects would benefit from high dopaminergic capacities [9]. The results of the Stroop task were similar but failed to reach statistical significance. However, due to our COMT and MAOA results, it is critical to explain the AP- $2 \beta$ effects through the possible modulation of the transcription of a single metabolic enzyme.

The VNTR MAOA polymorphism is functionally able to interfere with dopamine metabolism. Furthermore, it has previously been described to affect intelligence, aggression, risk taking, antisocial behavior, and treatment response [26-29]. In our sample, there were no significant effects on the cognitive performance parameters; however, we found a trend-like relationship. Interestingly, this relationship was counterintuitive: high-activity allele carriers showed slightly better cognitive results in the TMT-B and the Stroop task. Earlier studies have indicated that high-activity allele carriers should have lower dopamine levels and higher dopamine turnover. Nevertheless, our results were consistent with a previous report in healthy subjects that showed that MAOA-H carriers have a higher IQ compared with MAOA-L carriers [26].

Finally, we did not find any effects of COMT on executive functioning. There are some inconsistencies among previous investigations as to whether the COMT Val158Met polymorphism has a significant impact on individual cognitive performance. A meta-analysis by Barnett et al. [12] that included 46 studies concluded that the COMT polymorphism has only a small influence, if any, on cognitive performance. Our findings supported these results. It might be that the COMT polymorphism does not explain a significant proportion of individual performances while subjects are executing tasks that demand attention, set shifting, and working memory abilities. The absent or counterintuitive results in the present sample for the COMT Val158Met and VNTR MAOA polymorphisms do not support the idea that the AP- $2 \beta$ polymorphism exerts its effect on cognition simply through COMT and MAOA modulation, as previously thought. The direct effects of AP- $2 \beta$ on cognitive performance in the absence of any effects of COMT/MAOA suggest that the physiological mechanisms that are involved in the AP- $2 \beta$ association are not entirely based on the rate of dopamine metabolism but may also depend on the modulation of the transcription of various other gene products that are involved in signal transduction in the central nervous system.

The generalized linear model analysis, however, elucidated that decision bias $(\beta)$, in particular, was highly affected by multiple genotypes. All 3 polymorphisms showed significant main effects. Furthermore, the interaction of AP- $2 \beta$, MAOA, and COMT $\left(\chi^{2}=32.826, \mathrm{p}<\right.$ $0.005)$ showed a strong impact. These data suggested that the functional interplay between the corresponding gene products modulated the subject's decision making through dopamine metabolism. The decision bias provided a measure of whether decisions were generally more conservative or liberal. A score of 0 meant that there was no response bias, a positive score indicated a bias towards saying 'no', whereas a negative score indicated a bias towards saying 'yes' $[20,30]$. Looking at the results, simple interpretations of the high- or low-activity genotypes were not applicable as low MAOA subjects tended to show no bias and low COMT activity subjects showed a more conservative bias. Including the AP- $2 \beta$ promoter polymorphism in the statistical model provided a necessary factor for the interpretation of the model results. The MAOA-L/COMT (Val/Met)/AP-2 $\beta$ 5/5 carriers showed a more liberal bias compared to all other MAOA/COMT/ AP- $2 \beta 5 / 5$ groups. Because none of the 3 polymorphisms showed a liberal bias, it may be concluded that the interaction of the 3 genes turned the conservative or neutral biases into a more liberal one. These results need to be interpreted with caution due to the fact that some gene combinations had a very small group size $(n<2)$. Since some gene/allele frequencies are comparatively low, the presence of rare gene combinations is consequential. In fact, the actual group sizes (gene combinations) do not relevantly differ from the calculated and expected sizes. It may be speculated that the transcription rates of the AP$2 \beta$ polymorphism had the most prominent effect in the group with the highest dopamine availability (MAOA-L/ COMT-Val/Met). A study by Vernaleken et al. [31] supports this interpretation. In that positron emission tomography study, subjects with high baseline $\mathrm{D}_{2 / 3}$ receptor availability initially show better cognitive performance. However, when the dopamine system is challenged with ketamine, they show the highest level of drug-induced impairments. Thus, one might speculate that a subject with high levels of dopamine might be more vulnerable 
to manipulations of the structures, metabolism, or environmental stress. Nevertheless, it is more likely that these interactions involve complex neurobiological interactions of diverse structures rather than depict unidirectional and circumscribed mechanisms of the hierarchical pathway (i.e., transcription factor $\rightarrow$ MAOA/COMT transcription $\rightarrow$ MAOA/COMT activity $\rightarrow$ dopaminergic availability).

However, there is a negative correlation between neuroticism and decision bias $\beta$. Higher neuroticism is associated with less conservative bias/greater liberal bias. The decision bias $\beta$ is a value of the individual's weighing how to make the right decision. Subjects with more liberal bias tend to rather interpret a degraded stimulus as correct hit than to take the chance of omission. Subjects with more conservative bias, on the other hand, want to be rather sure that a degraded stimulus is unambiguously a correct target. The present correlational findings are in line with the literature: Burton et al. [32] found that neuroticism is negatively correlated with the decision bias $\beta$. Furthermore, they also found that in females the bias is negatively correlated with openness: higher scores of openness are associated with more liberal decisions. This association between these complex measures of personalities (mainly reflecting aspects of impulsivity) with distinct outcome parameters of a circumscribed cognitive task is astonishing but intuitive in its direction.

The current investigation also described a possible relationship between AP-2 $\beta / \mathrm{MAOA} / \mathrm{COMT}$ polymorphisms and personality factors. Based on the known literature, it is hypothesized that some subscales of personality questionnaires are associated with the dopamine system. Especially, novelty seeking and extraversion are suggested to be affected by dopamine transmission [3336]. These results, however, highly depend on the choice of assessment tools. In this sample, carriers of the $\mathrm{Val} / \mathrm{Val}$ allele of the COMT polymorphism showed significantly lower scores on the self-transcendence subscale in the character part of the TCI compared to Met/Met and Val/ Met carriers. Comings et al. [37] found that this subscale is sensitive to some genes that are involved in the dopaminergic system, including COMT. Similarly, in our sample, the Val/Val group scored higher on the NEO-FFI subscale of openness to experience, which has also been hypothesized to be associated with the dopaminergic system [38]. However, additional results of Deyoung et al. [39] present the opposite results of COMT and openness with Met/Met carriers having higher scores of openness compared to the $\mathrm{Val} / \mathrm{Val}$ group. Our results showed only a marginal difference between the 2 groups, even though this was a significant result. However, the previous study tested children who were aged between 8 and 13 years, who were non- or maltreated, and who grew up under low-income conditions [39]. In the present study, healthy female subjects (mainly students) were included, and this might suggest a bias of environmental or developmental factors. However, no socioeconomic data were systematically collected. Further investigations are needed to clarify this. Contrary to our expectation, there was no relationship between extraversion, novelty seeking, and the genotypes. This might be due to several reasons. As mentioned before, questionnaires differ in their ability to delineate personality traits complying with underlying neurobiological mechanisms. It is possible that the present choice of questionnaires which represents our standard protocol is not optimal to depict dopamine-related personality traits. Furthermore, it remains unclear whether the strict exclusion of male subjects impacts the results. Otherwise, there are previous studies which were also unable to find associations between the dopamine system (or AP-2 $\beta$ ) and novelty seeking $[36,40]$.

In the present population, there were no effects of AP$2 \beta$ on personality. Alternatively, a previous study of Damberg et al. [1] indicated that AP-2 $\beta$ influences anxietyrelated traits. Several reasons may account for this discrepancy. The sample sizes were comparatively low in both studies, and differences in inclusion criteria might be a relevant factor. Finally, Damberg et al. [1] used the Karolinska Scales of Personality, whereas, in this investigation, the NEO-FFI and the TCI were used. Another study of Damberg et al. [40] that used the TCI and the NEO-PI-R did not detect any associations either. A relevant impact of the AP- $2 \beta$ genotype on personality traits thus remains in question.

In summary, the AP- $2 \beta$ genotype is an important modulating factor of structures related to dopaminergic transmission. The data indicated that AP- $2 \beta$ had more influence on cognition than MAOA and COMT. Because MAOA and COMT showed little effect on cognition, we propose that AP- $2 \beta$ might influence cognition through pathways other than those that regulate MAOA and COMT.

\section{Acknowlegdements}

This work was supported by the German Research Foundation (DFG, VE 466/2-1, the IZKF Aachen, and IRTG 1328, International Research Training Group). Special thanks to Christine Frank.
88

Neuropsychobiology 2013;68:79-90 DOI: $10.1159 / 000350997$
Schabram/Eggermann/Siegel/Gründer/ Zerres/Vernaleken 


\section{Disclosure Statement}

Dr. Steven J. Siegel has received grant support from Astellas and AstraZeneca and has been a consultant to NuPathe, Lundbeck and Abbott regarding material that is unrelated to the work in the manuscript. Dr. Gerhard Gründer has served as a consultant for Bristol-Myers Squibb (New York, N.Y., USA), Cheplapharm (Greifswald, Germany), Eli Lilly (Indianapolis, Ind., USA), Lundbeck (Copenhagen, Denmark), Otsuka (Rockville, Md., USA), and
Servier (Paris, France). He has served on the speaker's bureau of Astra Zeneca, Bristol-Myers Squibb, Eli Lilly, Janssen Cilag, Otsuka, Pfizer, and Servier. He has received grant support from Alkermes, Bristol-Myers Squibb, Eli-Lilly, and Johnson \& Johnson. $\mathrm{He}$ is co-founder of Pharma-Image - Molecular Imaging Technologies GmbH (Germany). Dr. Klaus Zerres, Dr. Thomas Eggermann, Dr. Ingo Vernaleken, and Ina Schabram do not have any conflicts of interest to declare.

\section{References}

1 Damberg M, Garpenstrand H, Alfredsson J, Ekblom J, Forslund K, Rylander G, Oreland L: A polymorphic region in the human transcription factor AP-2beta gene is associated with specific personality traits. Mol Psychiatry 2000;5:220-224.

-2 Damberg M, Berggard C, Farde L, Sedvall GC, Jonsson EG: Transcription factor AP-2beta genotype, striatal dopamine $\mathrm{D} 2$ receptor density and cerebrospinal fluid monoamine metabolite concentrations in humans. J Neural Transm 2004;111:537-545.

3 Moser M, Ruschoff J, Buettner R: Comparative analysis of AP-2 alpha and AP-2 beta gene expression during murine embryogenesis. Dev Dyn 1997;208:115-124.

4 Damberg M: Transcription factor AP-2 and monoaminergic functions in the central nervous system. J Neural Transm 2005;112: 1281-1296.

5 Damberg M, Eller M, Tonissaar M, Oreland L, Harro J: Levels of transcription factors AP2alpha and AP-2beta in the brainstem are correlated to monoamine turnover in the rat forebrain. Neurosci Lett 2001;313:102-104.

6 Hensch T, Wargelius HL, Herold U, Strobel A, Oreland L, Brocke B: Electrophysiological and behavioral correlates of polymorphisms in the transcription factor AP-2beta coding gene. Neurosci Lett 2008;436:67-71.

7 Damberg M, Garpenstrand H, Hallman J, Oreland L: Genetic mechanisms of behavior - don't forget about the transcription factors. Mol Psychiatry 2001;6:503-510.

8 Mann JJ, Stanley M, Kaplan RD, Sweeney J, Neophytides A: Central catecholamine metabolism in vivo and the cognitive and motor deficits in parkinson's disease. J Neurol Neurosurg Psychiatry 1983;46:905-910.

-9 Vernaleken I, Buchholz HG, Kumakura Y, Siessmeier T, Stoeter P, Bartenstein P, Cumming P, Grunder G: 'Prefrontal' cognitive performance of healthy subjects positively correlates with cerebral FDOPA influx: an exploratory [18F]-fluoro-L-DOPA-PET investigation. Hum Brain Mapp 2007;28:931-939.

10 Vernaleken I, Kumakura Y, Buchholz HG, Siessmeier T, Hilgers RD, Bartenstein P, Cumming P, Grunder G: Baseline [18F]FDOPA kinetics are predictive of haloperi- dol-induced changes in dopamine turnover and cognitive performance: a positron emission tomography study in healthy subjects. Neuroimage 2008;40:1222-1231.

-11 Braskie MN, Wilcox CE, Landau SM, O’Neil JP, Baker SL, Madison CM, Kluth JT, Jagust WJ: Relationship of striatal dopamine synthesis capacity to age and cognition. J Neurosci 2008;28:14320-14328.

12 Barnett JH, Scoriels L, Munafo MR: Metaanalysis of the cognitive effects of the catechol-O-methyltransferase gene Val158/ 108Met polymorphism. Biol Psychiatry 2008; 64:137-144.

13 Barnett JH, Heron J, Ring SM, Golding J, Goldman D, Xu K, Jones PB: Gender-specific effects of the catechol-O-methyltransferase Val108/158Met polymorphism on cognitive function in children. Am J Psychiatry 2007; 164:142-149.

14 Farrell SM, Tunbridge EM, Braeutigam S, Harrison PJ: COMT Val(158)Met genotype determines the direction of cognitive effects produced by catechol-O-methyltransferase inhibition. Biol Psychiatry 2012;71:538-544.

15 Sheldrick AJ, Krug A, Markov V, Leube D, Michel TM, Zerres K, Eggermann T, Kircher $\mathrm{T}$ : Effect of COMT val158met genotype on cognition and personality. Eur Psychiatry 2008;23:385-389.

16 Bäumler G: Farbe-Wort-Interferenztest (FWIT) nach J.R. Stroop. Göttingen, Hogrefe, 1985.

17 Reitan RM, Wolfson D: Category test and trail making test as measures of frontal lobe functions. Clin Neuropsychol 1995;9:50-56.

18 Kathmann N, Wagner M, Satzger W, Engel RR: Vigilanzmessung auf Verhaltensebene: Der Continuous Performance Test-München (CPT-M); in Möller H-J, Engel RR, Hoff P (eds): Befunderhebung in der Psychiatrie: Lebensqualität, Negativsymptomatik und andere aktuelle Entwicklungen. Wien, Springer, 1996, pp 331-338.

19 Peterson WW, Birdsall TG, Fox WC: The theory of signal detectability. IEEE Trans Inform Theory 1954;PGIT-4:171-212.

20 Lee MD: BayesSDT: software for Bayesian inference with signal detection theory. Behav Res Methods 2008;40:450-456.
21 Borkenau P, Ostendorf F: NEO-Fünf-Faktoren-Inventar (NEO-FFI) nach Costa und McCrae (Handanweisungen). Göttingen, Hogrefe, 1993.

22 Goth K, Cloninger CR, Schmeck K: Das Temperament und Charakter Inventar Kurzversion für Erwachsene - TCI R Kurz. Frankfurt, Klinik für Psychiatrie und Psychotherapie des Kindes- und Jugendalters der J.W. GoetheUniversität Frankfurt, 2003.

23 Sabol SZ, Hu S, Hamer D: A functional polymorphism in the monoamine oxidase A gene promoter. Hum Genet 1998;103:273-279.

24 Rodriguez S, Gaunt TR, Day IN: HardyWeinberg equilibrium testing of biological ascertainment for Mendelian randomization studies. Am J Epidemiol 2009;169:505-514.

25 Chen J, Lipska BK, Halim N, Ma QD, Matsumoto M, Melhem S, Kolachana BS, Hyde TM, Herman MM, Apud J, Egan MF, Kleinman JE, Weinberger DR: Functional analysis of genetic variation in catechol-O-methyltransferase (COMT): effects on mRNA, protein, and enzyme activity in postmortem human brain. Am J Hum Genet 2004;75:807-821.

26 Yu YW, Yang CW, Wu HC, Tsai SJ, Hong CJ, Chen MC, Chen TJ: Association study of a functional MAOA-uVNTR gene polymorphism and personality traits in Chinese young females. Neuropsychobiology 2005;52:118121.

27 Meyer-Lindenberg A, Buckholtz JW, Kolachana B, A RH, Pezawas L, Blasi G, Wabnitz A, Honea R, Verchinski B, Callicott JH, Egan M, Mattay V, Weinberger DR: Neural mechanisms of genetic risk for impulsivity and violence in humans. Proc Natl Acad Sci USA 2006;103:6269-6274.

28 McDermott R, Tingley D, Cowden J, Frazzetto G, Johnson DD: Monoamine oxidase A gene (MAOA) predicts behavioral aggression following provocation. Proc Natl Acad Sci USA 2009;106:2118-2123.

29 Frydman C, Camerer C, Bossaerts P, Rangel A: MAOA-L carriers are better at making optimal financial decisions under risk. Proc Biol Sci 2011;278:2053-2059.

30 Stanislaw H, Todorov N: Calculation of signal detection theory measures. Behav Res Methods Instrum Comput 1999;31:137-149. 
-31 Vernaleken I, Klomp M, Möller O, Raptis M, Nagels A, Rösch F, Schaefer WM, Cumming P, Gründer G: Vulnerability to psychotogenic effects of ketamine is associated with elevated $\mathrm{D}(2 / 3)$-receptor availability. Int J Neuropsychopharmacol 2013;16:745-754.

- 32 Burton L, Pfaff D, Bolt N, Hadjikyriacou D, Silton N, Kilgallen C, Cofer J, Allimant J: Effects of gender and personality on the Conners Continuous Performance Test. J Clin Exp Neuropsychol 2010;32:66-70.

33 Cloninger CR: A unified biosocial theory of personality and its role in the development of anxiety states. Psychiatr Dev 1986;4:167-226.

34 Netter P: Dopamine challenge tests as an indicator of psychological traits. Hum Psychopharmacol 2006;21:91-99.
5 Stuettgen MC, Hennig J, Reuter M, Netter P: Novelty seeking but not BAS is associated with high dopamine as indicated by a neurotransmitter challenge test using mazindol as a challenge substance. Pers Individ Dif 2005;38:1597-1608.

36 Laakso A, Wallius E, Kajander J, Bergman J, Eskola O, Solin O, Ilonen T, Salokangas RK, Syvalahti E, Hietala J: Personality traits and striatal dopamine synthesis capacity in healthy subjects. Am J Psychiatry 2003;160: 904-910.

7 Comings DE, Gade-Andavolu R, Gonzalez N, Wu S, Muhleman D, Blake H, Mann MB, Dietz G, Saucier G, MacMurray JP: A multivariate analysis of 59 candidate genes in personality traits: the temperament and character inventory. Clin Genet 2000;58:375-385.
8 Deyoung CG, Peterson JB, Higgins DM: Sources of openness/intellect: cognitive and neuropsychological correlates of the fifth factor of personality. J Pers 2005;73:825-858.

39 Deyoung CG, Cicchetti D, Rogosch FA, Gray JR, Eastman M, Grigorenko EL: Sources of cognitive exploration: genetic variation in the prefrontal dopamine system predicts openness/intellect. J Res Pers 2011;45:364-371.

40 Damberg M, Berggard C, Mattila-Evenden M, Rylander G, Forslund K, Garpenstrand H, Bergman H, Gustavsson JP, Jonsson EG: Transcription factor AP-2beta genotype associated with anxiety-related personality traits in women. A replication study. Neuropsychobiology 2003;48:169-175. 